\title{
A MODIFIED K - $\varepsilon$ MODEL OF TURBULENCE
}

\author{
Showkat Jahan Chowdhury \\ Mechanical Engineering Department, Alabama A\&M University \\ P.O. Box 1163, Normal, AL 35762, U.S.A.
}

\begin{abstract}
In this paper, the parameters of a thermodynamically consistent $k-\varepsilon$ model of turbulence are first determined, and the numerical values of the various model coefficients are evaluated. Limiting flows of a decaying homogeneous turbulence, turbulent flow in the inertial sublayer, and known properties of purely diffusive turbulence are used for this purpose. The thermodynamically consistent model is then incorporated into an enhanced version of the TEACH computer code called STARPIC. Finally, the modified computational code is used to simulate the flow through a channel to assess its capability in predicting turbulent flows. The computational results are compared with available experimental data, and found to have reasonable matching. The flows are also simulated using standard $k-\varepsilon$ model, for comparison. It is observed that the present thermodynamically consistent modified $k-\varepsilon$ model predictions are better compared to the standard $k-\varepsilon$
\end{abstract} model predictions.

\section{INTRODUCTION}

Turbulence modeling has attracted considerable attention in recent years for solving practical turbulent flow problems. Simplified one and two-equation models and more elaborate stress transport models were developed in the past. Extensive reviews were provided in the literature ${ }^{1-6}$. The $\mathrm{k}-\varepsilon$ model has been extensively used for solving practical industrial turbulent flow problems ${ }^{1-3,7,8}$. One reason for the popularity of the two-equation model is that it can be conveniently accommodated in the computer codes for solving the Navier-Stokes equation. However, several questions concerning the accuracy of the standard $\mathrm{k}$ $-\varepsilon$ model have been raised. Especially, the closure assumptions involved in the $\varepsilon$-equation are not considered to be satisfactory ${ }^{9,10}$

The averaged form of the Clausius-Duhem inequality was derived by $\mathrm{Ahmadi}^{6}$ and its consequences on turbulence modeling were discussed. Based on the thermodynamical arguments a two-equation turbulence model was developed which resembles the $\mathrm{k}-\varepsilon$ model $^{6,11}$. In the new model, the k-equation remains unchanged, while the $\varepsilon$-equation is quite different from the standard one. That is, the standard $\varepsilon$-equation is not consistent with the second law of thermodynamics, while the new $\varepsilon$ equation is consistent.

The purpose of this study is to first determine the parameters for the above model equations. Limiting flows of a decaying homogeneous turbulence and turbulent flow in the inertial sublayer are used for this purpose. The new model is then used to study a purely diffusive turbulence in the absence of a mean velocity field. The experimental results of Hopfinger and Toly ${ }^{12}$ are used to evaluate the values of some constants. Finally, the new model is incorporated into a computer code, and is used to simulate the flow through a channel to assess its capability in computing complex turbulent flows.

\section{BASIC EQUATIONS}

The equations governing the mean turbulent motion of an incompressible fluid are given as ${ }^{6,11}$,

$$
\begin{aligned}
& \dot{v}_{i, i}=0 \\
& \rho \dot{v}_{i}=-P_{, i}+\left[\left(\mu+\mu^{T}\right)\left(v_{i, j}+v_{j, i}\right)\right]_{, j}+\rho f_{i}
\end{aligned}
$$

$$
\begin{gathered}
\rho \dot{C}=\left[\left(\kappa+\frac{\mu^{T}}{\sigma^{\theta}}\right) \theta_{, i}\right]_{, i}+\mu\left(v_{i, j}+v_{j, i}\right) v_{j, i}+\rho \varepsilon+\rho r \\
\rho \dot{k}=\left[\left(\mu+\frac{\mu^{T}}{\sigma^{\kappa}}\right) k_{, i}\right]_{, i}+\mu^{T}\left(v_{i, j}+v_{j, i}\right) v_{j, i}-\rho \varepsilon \\
\dot{\rho \varepsilon}=\varepsilon\left[\frac{1}{\varepsilon}\left(\mu+\frac{\mu^{T}}{\sigma^{\varepsilon}}\right) \varepsilon_{, i}\right]_{, i}+C^{\varepsilon 1} \frac{\mu^{T} \varepsilon}{k}\left(v_{i, j}+v_{j, i}\right) v_{j, i}(5) \\
+C^{\varepsilon 3}\left(\mu+\frac{\mu^{T}}{\sigma^{\kappa}}\right) \frac{\varepsilon}{k^{2}} k_{, i} k_{, i}-\rho C^{\varepsilon 2} \frac{\varepsilon^{2}}{k}
\end{gathered}
$$

Equations (1) - (4) are the statements of conservation of mass, balance of linear momentum, conservation of thermal energy, and conservation of turbulence fluctuation energy. Eq. (5) which is a transport equation for dissipation rate was derived from the entropy inequality ${ }^{6,11}$. In these equations $\rho$ is the mass density, $v_{i}$ is the mean velocity, $f_{i}$ is the body force per unit mass, $\mu$ is the coefficient of viscosity, $\mu^{T}$ is the coefficient of turbulent (eddy) viscosity, $P$ is the total mean pressure, $C$ is the averaged heat capacity, $\theta$ is the modified averaged temperature, $\kappa$ is the heat conductivity, $\sigma^{\theta}$ and $\sigma^{\mathrm{k}}$ are the turbulent Prandtl

\begin{tabular}{|c|c|}
\hline \multicolumn{2}{|c|}{ Nomenclature } \\
\hline $\mathrm{H}$ & half-width of channel \\
\hline $\mathrm{k}$ & turbulent kinetic energy \\
\hline $\mathrm{P}$ & total mean pressure \\
\hline $\operatorname{Re}$ & Reynolds number \\
\hline$v_{i}$ & mean velocity \\
\hline $\mathrm{U}$ & mean axial velocity \\
\hline $\mathrm{U}_{\mathrm{c}}$ & centerline mean velocity \\
\hline $\mathrm{u}^{*}$ & shear velocity \\
\hline $\mathrm{y}$ & distance from channel wall \\
\hline$\varepsilon$ & dissipation rate of turbulence kinetic energy \\
\hline$\mu_{T}$ & coefficient of viscosity \\
\hline$\mu^{\mathrm{T}}$ & coefficient of turbulent or eddy viscosity \\
\hline$\rho_{1}$ & mass density \\
\hline$\sigma_{\varepsilon}^{\mathrm{k}}$ & turbulent Prandtl number corresponding to $k$ \\
\hline$\sigma^{\varepsilon}$ & turbulent Prandtl number corresponding to $\varepsilon$ \\
\hline
\end{tabular}
numbers, $\varepsilon$ is the dissipation rate, $r$ is the internal heat source per unit mass, $k$ is the fluctuation kinetic energy and $\sigma^{\varepsilon}$ is a turbulent Prandtl number corresponding to $\varepsilon$. In these equations regular tensor notation with Latin subscripts is employed. Thus, indices after a comma denote 
partial derivatives and a dot on the top of a letter stands for material derivative with respect to the mean flow.

The constants $C^{\varepsilon 1}, C^{\varepsilon 2}$ and $C^{\varepsilon 3}$ in Eq. (5) are restricted to,

$\frac{1}{\alpha} \geq C^{\varepsilon 1} \geq 0, C^{\varepsilon 2} \geq \frac{1}{\alpha}, \frac{1}{\alpha} \geq C^{\varepsilon 3} \geq 0, \alpha>0$

The eddy viscosity is given as,

$\mu^{T}=\rho C^{\mu} \frac{k^{2}}{\varepsilon}$

where $C^{\mu}$ is a constant

Equations (1) - (5) together with Eq. (7) form a complete set of seven transport equations for determining the seven unknowns $v_{i}, k, \theta, \varepsilon$ and $P$. These equations are almost identical to those of the standard $\mathrm{k}-\varepsilon$ model $^{3}$, except Eq. (5) which is somewhat different from the common $\varepsilon$-equation. The differences appear in the diffusion and destruction terms.

\section{CONSTRAINTS ON THE MODEL PARAMETERS}

The developed model involves several coefficients which must be estimated. Known properties of simple turbulent flows may be used to relate these coefficients.

Decay Behind a Grid: Consider a homogeneous decaying turbulence behind a grid. For small axial diffusion. Eqs. (4) and (5) become,

$U_{0} \frac{d k}{d x}=-\varepsilon$

$U_{0} \frac{d \varepsilon}{d x}=-C^{\varepsilon 2} \frac{\varepsilon^{2}}{k}$

where $U_{o}$ is the constant axial mean velocity. Eqs. (8) and (9) accept the following solutions,

$k \approx x^{\frac{1}{\left(1-C^{\varepsilon 2}\right)}}, \quad \varepsilon \approx x^{C^{\varepsilon 2} /\left(1-C^{\varepsilon 2}\right)}$

These results are identical to that of the standard $\mathrm{k}-\varepsilon$ model. Comparing Eq. (10) with the experimental data, it follows that ${ }^{1,3} C^{e 2} \approx 2$. The values of $C^{e 2}=1.9$ is recommended in more recent works ${ }^{2,4,13}$.

Inertial Sublayer: In the inertial sublayer close to the wall, $k$ becomes a constant, the production is equal to dissipation and Eqs. (4) and (5) may be restated as,

$\mu^{T}\left(\frac{d v_{1}}{d y}\right)^{2}-\rho \varepsilon=0$

$k \frac{d}{d y}\left(\frac{\mu^{T}}{\varepsilon \sigma^{\varepsilon}} \frac{d \varepsilon}{d y}\right)+C^{\varepsilon 1} \mu^{T}\left(\frac{d v_{1}}{d y}\right)^{2}-\rho C^{\varepsilon 2} \varepsilon=0$

The turbulent flow in the inertial sublayer is characterized by a velocity scale $u^{*}$ (shear velocity) and a length scale $y$ (distance from the wall). The flow field parameters are then given as ${ }^{1,14}$,

$v_{1}=\frac{u^{*}}{\kappa} \ln y+B, \quad k=\frac{u^{* 2}}{\left(C^{\mu}\right)^{\frac{1}{2}}}, \quad \varepsilon=\frac{u^{* 3}}{\kappa y}, \quad \mu^{T}=\rho \kappa u^{*} y$,

where $\kappa$ is the Karman constant. Using Eq. (13), Eq. (11) is now identically satisfied. If $\sigma^{\varepsilon}$ be treated as a constant, Eq. (12) accepts the solution given by Eq. (13) if,

$C^{\mathrm{el}}=C^{\mathrm{e} 2}$

But with Eq. (14), it may be shown that the model predicts the existence of an equilibrium homogeneous uniform shear field. However, the existing experimental data show that such flows are continuously evolving ${ }^{15}$. To avoid Eq. (14), while still ensuring that the model accepts the inertial sublayer solutions given by Eq. (13), it is assumed that,

$\sigma^{\varepsilon}=\sigma^{\varepsilon 0} /\left[1-\beta \ln \frac{\mu^{T}}{\mu}\right]$

where $\beta$ and $\sigma^{\varepsilon o}$ are some constants. Using Eq. (15), it follows that Eq. (13) satisfies Eq. (12) if,

$C^{\varepsilon 2}-C^{\varepsilon 1}=\frac{\beta \kappa^{2}}{\sigma^{\varepsilon 0}\left(C^{\mu}\right)^{\frac{1}{2}}}$

Diffusive Homogeneous Turbulence: $\operatorname{Sonin}^{16}$ used the data of Thompson et al. ${ }^{17}$ and Hopfinger et al. ${ }^{12}$ for a purely diffusive homogeneous turbulence (with zero mean velocity) to calibrate the constants of the standard $k-\varepsilon$ model. He also noted an inconsistency in the commonly used values of the constants.

In the absence of a mean velocity field, Eqs. (4) and (5) may be restated as,

$\frac{d}{d x}\left(\frac{\mu^{T}}{\sigma^{k}} \frac{d k}{d x}\right)-\rho \varepsilon=0$

$\frac{d}{d x}\left(\frac{1}{\varepsilon} \frac{\mu^{T}}{\sigma^{\varepsilon}} \frac{d \varepsilon}{d x}\right)+C^{\varepsilon 3} \frac{\mu^{T}}{\sigma^{k} k^{2}}\left(\frac{d k}{d x}\right)^{2}-\rho C^{\varepsilon 2} \frac{\varepsilon}{k}=0$

Eqs. (17) and (18) imply that the diffusions of $k$ and $\varepsilon$ must be, respectively, balanced by the dissipation rate and net destruction of $\varepsilon$. For $\sigma^{\mathrm{k}}$ constant and $\sigma^{\varepsilon}$ given by Eq. (15), from Eq. (17) the solutions are found as,

$k=K_{0} x^{-n}, \varepsilon=E_{0} x^{-\left(\frac{3 n}{2}+1\right)}$

where $K_{o}$ and $E_{o}$ are some constants and their relation is also obtained as,

$E_{0}=0.7348 K_{0}^{\frac{3}{2}}$

Substituting Eq. (15) and Eq. (19) into Eq. (18) we get for $\mathrm{n}=2$,

$C^{\varepsilon 2}=\frac{2 \sigma^{k}}{3 \sigma^{\varepsilon 0}}\left(1-\beta \ln \frac{\mu^{T}}{\mu}\right)+\frac{2}{3} C^{\varepsilon 3}$

Here we have taken $n=2$, as experimental data of Hopfinger and Toly ${ }^{12}$ supports $\mathrm{n}=2$. Eqs. (15), (16) and (21) then relates the parameters of the model. Now let,

$C^{\varepsilon 1}=1.67, \quad C^{\varepsilon 2}=1.92, \quad C^{\varepsilon 3}=1.92, \quad \kappa=0.42$,

$C^{\mu}=0.09, \sigma^{k}=1.0$

Then from Eqs. (21) and (16) we obtain,

$\sigma^{\varepsilon 0}=0.257, \beta=0.109$

The constants in Eq. (23) are found for $\mu^{\mathrm{T}} / \mu=1000$. From Eq. (21), the value of $\mathrm{C}^{\mathrm{\varepsilon} 3}$ is obtained as,

$$
\begin{gathered}
C^{\varepsilon 3}=\frac{3}{2} C^{\varepsilon^{2}}-\frac{\sigma^{k}}{\sigma^{\varepsilon 0}}\left(1-\beta \ln \frac{\mu^{T}}{\mu}\right) \\
\text { when } \frac{\mu^{T}}{\mu} \leq 1000 \\
C^{\varepsilon 3}=1.92 \text { when } \frac{\mu^{T}}{\mu} \geq 1000
\end{gathered}
$$

These values are somewhat different from those used in the standard $\mathrm{k}-\varepsilon$ model. The coefficients satisfy the restrictions of Eq. (6).

An enhanced version of the TEACH computer code called STARPIC is modified to account for the requirements of the above turbulence model based on the second law of thermodynamics. The computational model 


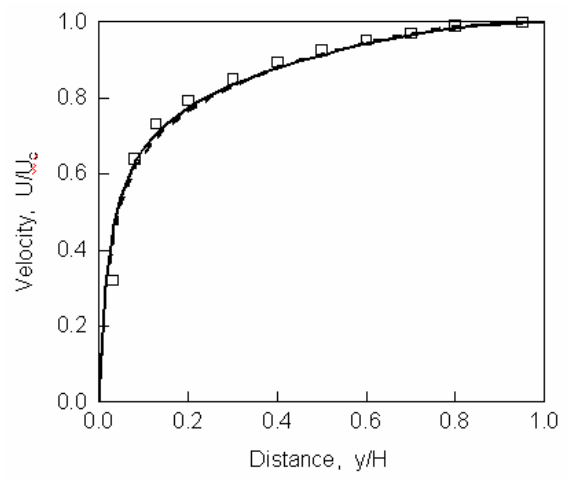

Figure 1: Comparison of dimensionless mean velocity profiles: $\square$, experimental data of Eckelmann; , present model; -----, standard k- $\varepsilon$ model.

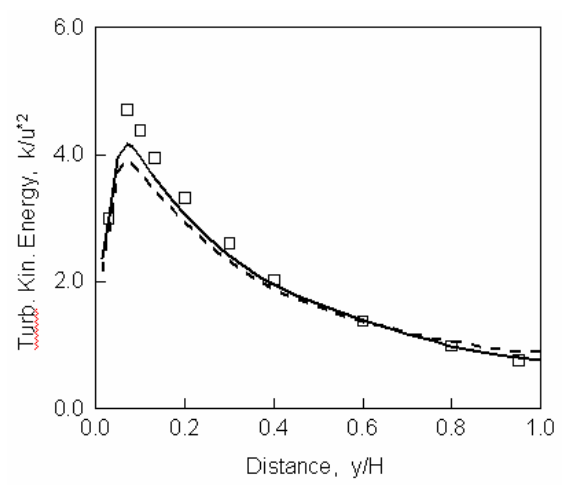

Figure 2: Comparison of dimensionless turbulent kinetic energy profiles: $\square$, experimental data of Kreplin et al.; present model prediction; -----, standard $\mathrm{k}-\varepsilon$ model.

is then used to simulate the turbulent flow through a channel. The modified computational code uses a staggered mesh finite volume method to discretize the governing differential equations. The computational domain is divided by a $46 \times 34$ non-uniform grid with finer spacing in the regions of large spatial gradients. The above differential equations are integrated over their appropriate staggered control volumes and discretized using a hybrid differencing scheme $^{18}$. The discretized equations with boundary condition modifications are solved using the SIMPLE $^{19}$ and TDMA algorithm. Here, semi-implicit lineby-line relaxation method is employed to obtain converged solutions iteratively. Under relaxation factors are also used to promote computational stability. The results of computations are discussed in the subsequent sections.

\section{CHANNEL FLOW}

Turbulent flow through a channel has served as a primary benchmark for assessing the performance of various turbulence models in predicting turbulent flows. Using hot-film probes, the mean flow properties in a fully developed turbulent flow of oil in a channel were measured experimentally by Eckelmann ${ }^{20}$ and Kreplin and Eckelmann $^{21}$. In these experiments a $22 \mathrm{~cm}$ wide and 79 $\mathrm{cm}$ deep channel was used so that the flow may be considered as being roughly two-dimensional. The kinematic viscosity of the oil was $0.06 \mathrm{~cm}^{2} / \mathrm{s}$. Eckelmann ${ }^{20}$ performed the experiment at Reynolds number of 8200 based on the channel width and centerline mean velocity. A Reynolds number of 7700 was used in the study of Kreplin and Eckelmann ${ }^{21}$.

The computational code which incorporates the new modified $\mathrm{k}-\varepsilon$ model is used to simulate the turbulent channel flow of oil at a Reynolds number of 7700 , for the above experimental conditions. The flow was also simulated using standard $\mathrm{k}-\varepsilon$ model for comparison. The predictions of the new model are compared with the experimental results ${ }^{20,21}$ and those by the standard $\mathrm{k}-\varepsilon$ model in Figs. 1 - 2. In these figures, the solid and dashed lines correspond to the predictions of the present model and those of the standard $\mathrm{k}-\varepsilon$ model, respectively, and the boxes correspond to the experimental data ${ }^{20,21}$.

The variation of the nondimensional mean axial velocity $U / U_{c}$ across the channel as predicted by the present model is shown in Fig. 1. Here, $U_{c}$ is the centerline mean velocity, $y$ is the distance from the channel wall and $H$ is the half-width of the channel. The velocity distribution obtained using the standard $\mathrm{k}-\varepsilon$ model and the experimental data of Eckelmann ${ }^{20}$ for Reynolds number of 8200 are also shown in this figure for comparison. It is observed that the predicted mean velocity distribution by both models is in good agreement with the experimental data, while the modified $\mathrm{k}-\varepsilon$ model doing slightly better.

Fig. 2 shows the distribution of the predicted turbulence kinetic energy across the channel by both the new modified $\mathrm{k}-\varepsilon$ model and standard $\mathrm{k}-\varepsilon$ model, along with the experimental data of Kreplin and Eckelmann ${ }^{21}$. The turbulence kinetic energy $k$ is nondimensionalized with the aid of the shear velocity $u^{*}$. As mentioned above, $y$ is the distance from the channel wall and $H$ is the halfwidth of the channel. The steep gradient of the velocity profile near the wall means shearing of the fluid layers which generates large amount of turbulence kinetic energy. But in the regions very close to the wall, dissipation rates are high as they are inversely proportional to the length scale (distance from the wall), and so turbulence kinetic energy profile very close to the wall goes down. It is observed that the predictions of the present model are in better agreement with the experimental data in comparison to those by the standard $\mathrm{k}-\varepsilon$ model.

The presented results show that the thermodynamically consistent modified $\mathrm{k}-\varepsilon$ model is capable of predicting the features of turbulent flow through a channel.

\section{CONCLUSION}

Known properties of simple turbulent flows are first used to relate the coefficients of the thermodynamically consistent modified $\mathrm{k}-\varepsilon$ turbulence model. The numerical values of the model parameters are then evaluated.

An enhanced version of the TEACH computer code called STARPIC, is modified to include this $\mathrm{k}-\varepsilon$ turbulence model based on the second law of thermodynamics. The modified computational code is then used to simulate the turbulent flow through a channel, and the results are compared with available experimental data. It is found that the model predictions have reasonable matching with the experimental data. The flow is also simulated using standard $\mathrm{k}-\varepsilon$ model, for comparison. It is observed that the present thermodynamically consistent modified $\mathrm{k}-\varepsilon$ model predictions are better compared to the standard $\mathrm{k}-\varepsilon$ model predictions.

Joumal of Mechanical Engineering, vol. ME38, Dec. 2007 


\section{REFERENCES}

1. Launder, B.E. and Spalding, D.B., "Mathematical Models of Turbulence", Academic Press, New York (1972).

2. Rodi, W., "Examples of Turbulence Models for Incompressible Flows", AIAA J., Vol.20, pp. 872-889 (1982).

3. Jones, W.P. and Launder, B.E., "The Prediction of Laminarization with a Two-equation Model of Turbulence", Int. J. Heat Mass Transfer, Vol. 15, pp. 301-314 (1972).

4. Hanjalic, K. and Launder, B.E., "Contribution Toward a Reynolds-Stress Closure for Low Reynolds-Number Turbulence", J. Fluid Mech., Vol. 74, pp. 593-610 (1976).

5. Newman, G.R., Launder, B.E. and Lumley, J.L., "Modelling the Behaviour of Homogeneous Scalar Turbulence", J. Fluid Mech., Vol. 111, pp. 217-232 (1981).

6. Ahmadi, G., "On Thermodynamics of Turbulence", Bulletin of the American Physical Society, Vol. 29, pp. 1529 (1984).

7. Launder, B.E., and Spalding, D.B., "The Numerical Computation of Turbulent Flows", Computer Methods in Applied Mechanics and Engineering, Vol. 3, pp. 269-289 (1974).

8. Busnaina, A.A. and Lilley, D.G., Chemical Engineering Communications, Vol. 26, pp. 89-104 (1984).

9. Lumley, J.L., "Turbulence Modeling”, Journal of Applied Mechanics, Trans. ASME, Vol. 50, pp. 10971103 (1983).

10. Zeman, O., "Progress in the Modeling of Planetary Boundary Layers", Annual Review of Fluid Mechanics, Vol. 13, (ed. M. VanDyke, V. Wehausen and J.L. Lumley), Palo Alto, CA, pp. 253-272 (1981).
11. Chowdhury, S.J., "Thermodynamically Consistent Rate-Dependent Models for Turbulence", Ph.D. Thesis, Clarkson University, New York (1990).

12. Hopfinger, E.J. and Toly, J.A., "Spatially Decaying Turbulence and its Relation to Mixing Across Density Interfaces", J. Fluid Mech., Vol. 78, pp. 155-175 (1976).

13. Bradshaw, P., Cebeci, T. and Whitelaw, J.H., "Engineering Calculation Methods for Turbulent Flow”, Academic Press, New York (1981).

14. Tennekes, H. and Lumley, J.L., "A First Course in Turbulence", MIT Press, Massachusetts (1981).

15. Gence, J.N., "Homogeneous Turbulence", Annual Review of Fluid Mechanics, Vol. 15, pp. 201-222 (1983).

16. Sonin, A.A., "Calibration of the $\mathrm{k}-\varepsilon$ Turbulence Model for the Diffusion of Turbulence", Phys. Fluids, Vol. 26, p. 2769 (1983).

17. Thompson, S.M. and Turner, J.S., "Mixing Across an Interface Due to Turbulence Generated by an Oscillating Grid", J. Fluid Mechanics, Vol. 67, pp. 349-368 (1975).

18. Patankar, S.V., "Numerical Heat and Fluid Flow", McGraw-Hill, New York (1980).

19. Gosman, A.D. and Pun, W.M., "Calculation of Recirculating Flows", Report No. HTS/74/12, Dept. of Mechanical Engineering, Imperial College, London (1974).

20. Eckelmann, H., "The Structure of the Viscous Sublayer and the Adjacent Wall Region in a Turbulent Channel Flow", J. Fluid Mechanics, Vol. 65, pp. 439459 (1974).

21. Kreplin, H. and Eckelmann, H., "Behavior of the Three Fluctuating Velocity Components in the Wall Region of a Turbulent Channel Flow", Phys. Fluids, Vol. 22, pp. 1233-1239 (1979). 